\title{
Téoros
}

Revue de recherche en tourisme

\section{Les effets à court et long terme sur le tourisme des célébrations du 350e anniversaire de Montréal en 1992}

\section{Pierre Labrie}

Volume 10, numéro 3, novembre 1991

Destination Montréal : d'hier à demain

URI : https://id.erudit.org/iderudit/1079202ar

DOI : https://doi.org/10.7202/1079202ar

Aller au sommaire du numéro

Éditeur(s)

Université du Québec à Montréal

ISSN

0712-8657 (imprimé)

1923-2705 (numérique)

Découvrir la revue

Citer cet article

Labrie, P. (1991). Les effets à court et long terme sur le tourisme des célébrations du 350e anniversaire de Montréal en 1992. Téoros, 10(3), 40-43. https://doi.org/10.7202/1079202ar d'utilisation que vous pouvez consulter en ligne. 


\section{Les effets à court et long terme sur le tourisme des célébrations du $350 \mathrm{e}$ anniversaire de Montréal en 1992}

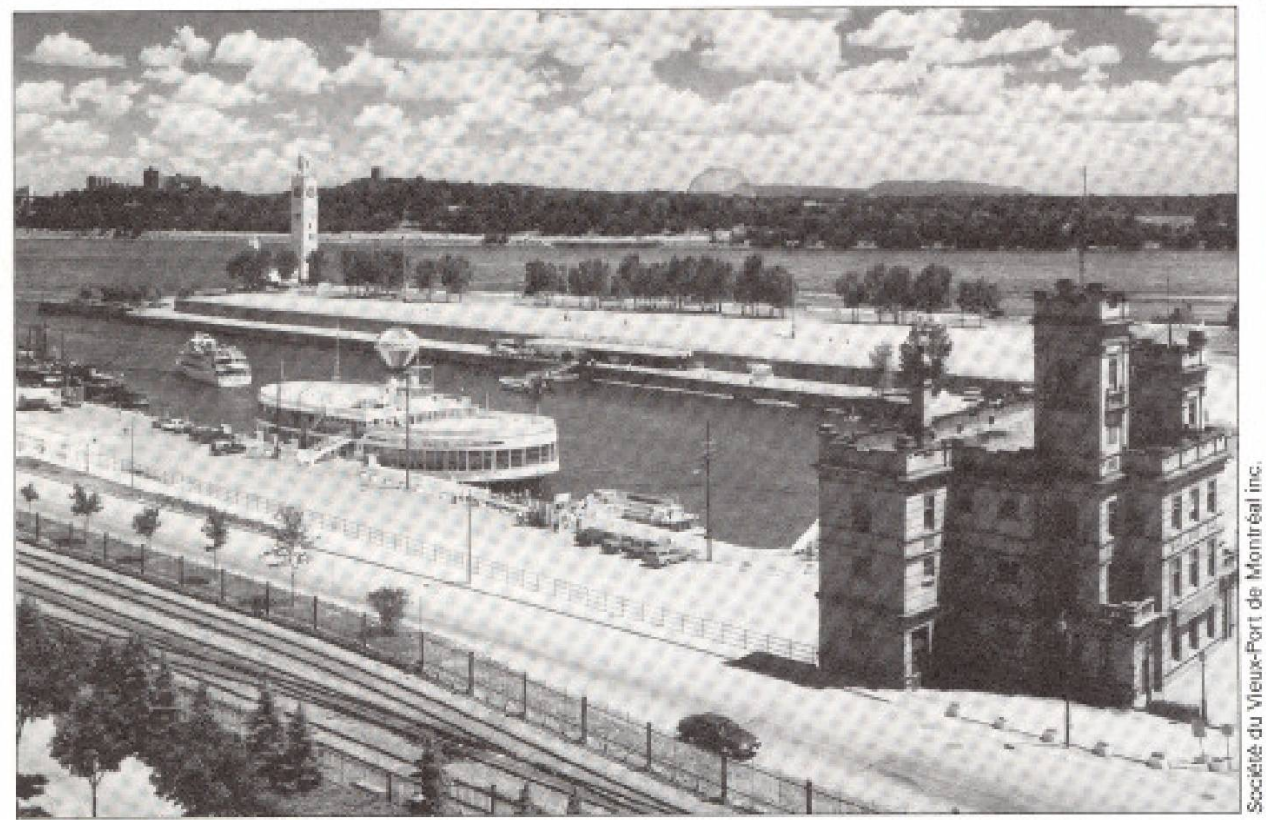

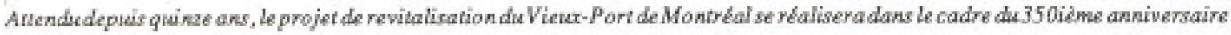
de Montríd

\section{Contexte}

Àquelques mois de l'inauguration des célébrations du 350 e anniversaire de Montréal le 15 mai 1991, on peut $s$ 'interroger sur les effets durables des sommes consacrées à l'organisation de ces célébrations. Ces questions sont bien légitimes, comple tenu que nous ne vivons pas présentement dans un contexte où nous pouvons investir et mener de front tous les projets nécessaires pour le mieux-être de nos concitoyenset pourle développement de nos divers secteurs d'activités.

Au cours des prochains mois, des équipes de spécialistes procéderont à l'évaluation des effets à courtet long terme des célébrations. Diversesapproches serontretenues, plusieurs techniques utilisées et de nombreux intervenants mis à contribution. Ce que l'on re-

\footnotetext{
Monsieur Pierre Labrie était jusqu'en septembre 1991 assistant-directeur aux Affaires publiques de la Vile de Montrubal. II etait responsable entre autres des relations entre la Ville de Montreal et la Corporation du 350 e anniversaire de Montréal. Pierre Labrie est maintenant directeur de f'office du tourisme et des congrès de la communauté urbaine de Québẹ (OTCCUO).
}

En pratique, toutes les etudes qui seront réaliséespermettront de confirmer ounon les effets positifs ou négatifs associés aux célébrations. Quelles que soient les techniques utilisées et l'ampleur des moyens consacrés, lescommentaires et appréciations permettront d'évaluer l'événement dans sa globalité.

A partir des données recueillies au cours des deux dernières années, il est toutefois possible d'identifier avec précision certains éléments contributifs à l'organisation et à la réalisation des célébrations du $350 \mathrm{e}$. Ces bléments contributifs sont de quatre ordres:

\section{dépenses d'organisation; dépenses de production; dépenses d'immobilisations - directement liées; . indirectement associées.}

Ces dépenses totalisent plus de $400 \mathrm{M} \$$ dont. prés de $200 \mathrm{M} \$$ peuvent être associés directement au concept développé pour souligner en 1992 le 350 e anniversaire de la fondation de Montréal. Le solde, environ 200 $\mathrm{M} \$$, peut yễtreassocié indirectement, compte tenu que la natureetles dates de réalisation de ces travaux coincident plus ou moins avec la période des célébrations.

\section{Dépenses d'organisation} visiteurs, sur notre propre comportement en terme psychologique et même sur le climat politique qui colore notre vie en société.

Compte tenuque lademarche scientifique de l'évaluation des célébrations du 350 e anniversaire n'a pas encore étế initiếe et que les données nécessaires à ce travail n'ont pas encoreêté entièrementcolligées, ilest impossible de présenter avec exactitude un tableau des effets anticipés par les célébrations du 350 e. Toutefois, au plan théorique, la grille d'analyse-synthèse développée par monsieur Brent Ritchie, professeur à l'Universitê de Calgary, Alberta, et publiée en 1984 dans le T.T.R.A. Journal, présente três bien la typologie des impacts et des effets des événements majeurs (voir tableau 1).
Le budget d'organisation des célébrations du 350 e anniversaire sera de l'ordre de $46 \mathrm{M} \$$ dont environ $20 \mathrm{M} \$$ associes aux frais de gestion, de developpement et de promotion des célébrations et environ $25 \mathrm{M} \$$ au titre de la programmation directe et déléguée des activités prévuesen 1992. Cebudgetprovient de quatre sources de financement: le gou= vernement du Québec pour $10 \mathrm{M}$ \$; le gouvernement du Canada pour $10 \mathrm{M} \$$; la Ville de Montréal pour 14,1 M \$ et, enfin, le secteur privé pour une somme d"environ 12 M \$; ce dernier volet inclut les revenus autogénérés par l'organisation de certaines activités. 


\section{Dépenses de production}

La Corporation Montréal 1992 investit une somme d'environ $25 \mathrm{M}$ \$ en programmation d'activités et d'événements qui seront réalisés au cours de 1992. La moitié de cette somme a été engagée directement par la Corporation à la production d'événements dont elle assume le plein contrôle.

À titre d'exemples, production d'émissions de télévision pour lancer la programmation des célébrations, développement d'une exposition multi-média en collaboration avec plusieurs musées montréalaisetanimation de lieux et de places publiques. Au total, de 10 M à $12 \mathrm{M}$ \$ seront donc consacrés à une programmation nouvelle et originale identifiée clairement aux événements de 1992.

Le solde du budgetconsacré à la programmation, $12 \mathrm{Mà} 13 \mathrm{MS}$, a été engagé auprès de producteurs délégués et partenaires du secteur privé qui eux-mêmes réalisent chaque année un ou plusieurs événements spéciaux. A ce titre, les promoteurs du Festival de Jazz de Montreal, du Festival Juste pour Rire, du Festival des Films du Monde, recevront en 1992 une contributionde laCorporationetce, afin de créer un volet Célébrations du $350 e$ anniversaire à l'intérieur de leur programmation.Autrement dit, les sommes consacrées àce volet de production servent de levier pour créer une valeur ajoutée à l'intérieur d'événementsetactivitésqui se réalisent déjà dans la région de Montréal.

En complément des méga-événements du $350 \mathrm{e}$, la Corporation a sollicité un engagement communautaire important et tenté d'associer, dans tous les arrondissements de Montréal, la population à la célébration du 350 e anniversaire. Il en découle plus de 160 événements nouveaux à caractère multiculturel, communautaire, non récurrents qui se dérouleront spécifiquement en 1992 dans le cadre du $350 \mathrm{e}$. Ces événements sont, en partie, financés par la Corporation, le solde des ressources requises provenant, dans bien des cas, de contributions du secteur privé ou de la population. Donc, une partie dece $12 \mathrm{M}$ $\$$ en frais de production a définitivement un effet multiplicateur et générateur de produits nouveaux exclusifs à l'année 1992.

Seule une évaluation postérieure à la réalisation de ces activités permettra de confirmer ou non la valeur ajoutée réelle en terme de nouveaux dollars consacrés à la production.

\begin{tabular}{|c|c|c|}
\hline \multicolumn{3}{|c|}{$\begin{array}{l}\text { TABLEAU } 1 \\
\text { Types de répercussions d'ếnénements de grande envergure }\end{array}$} \\
\hline \multicolumn{3}{|c|}{ Manifestations } \\
\hline Répercussions & positives & négatives \\
\hline Économiques & $\begin{array}{l}\text { Hausse des dépenses. } \\
\text { Création d'emplois. }\end{array}$ & $\begin{array}{l}\text { Hausse des prix pendant } \\
\text { l'événement. } \\
\text { Spéculation immobilière. }\end{array}$ \\
\hline Touristiques/commerciales & $\begin{array}{l}\text { Accroissement de la sensi- } \\
\text { bilisation au potentiel } \\
\text { touristique de la région. } \\
\text { Mise en valeur du potentiel } \\
\text { commercial et d'investis- } \\
\text { sement de la région. }\end{array}$ & $\begin{array}{l}\text { Réputation en jeu en cas } \\
\text { d'installations ou de méthodes } \\
\text { inappropriés. } \\
\text { Réticences des entreprises } \\
\text { existantes face à une eventuelle } \\
\text { nouvelle compétition pour la } \\
\text { main-d'oeuvre locale et les } \\
\text { subventions gouvemementales. }\end{array}$ \\
\hline Physiques & $\begin{array}{l}\text { Construction de nouvelles } \\
\text { installations. } \\
\text { Amélioration de } \\
\text { l'infrastructure locale. }\end{array}$ & $\begin{array}{l}\text { Dangers pour l'environnement. } \\
\text { Surpopulation. }\end{array}$ \\
\hline Socio-culturelles & $\begin{array}{l}\text { Accroissement du niveau } \\
\text { permanent d'intérết et de } \\
\text { participation locaux au type } \\
\text { d'activité associée a } \\
\text { l'événement. } \\
\text { Renforcement des traditions } \\
\text { et des valeurs régionales. }\end{array}$ & $\begin{array}{l}\text { Commercialisation d'activités } \\
\text { d'ordre personnel ou privé. } \\
\text { Modification de la nature de } \\
\text { l'événement/de l'activité pour } \\
\text { des fins touristiques. }\end{array}$ \\
\hline Psychologiques & $\begin{array}{l}\text { Plus grande fiente locale et } \\
\text { meilleur esprit. } \\
\text { communautaire. } \\
\text { Accroissement de la sensi- } \\
\text { bilisation aux perceptions } \\
\text { non locales. }\end{array}$ & $\begin{array}{l}\text { Tendance à des attitudes } \\
\text { défensives envers les régions } \\
\text { hötes. } \\
\text { Forte possibilité de } \\
\text { malentendus menant à une } \\
\text { certaine hostilité envers } \\
\text { l'hôte ou le visiteur. }\end{array}$ \\
\hline _Politiques & $\begin{array}{l}\text { Plus forte reconnaissance } \\
\text { internationale de la région } \\
\text { et de ses valeurs. } \\
\text { Propagation des valeurs } \\
\text { politiques véhiculées par } \\
\text { le gouvemement/la } \\
\text { population. }\end{array}$ & $\begin{array}{l}\text { Exploitation économique de la } \\
\text { population locale pour } \\
\text { satisfaire les ambitions de } \\
\text { l'elite politique. } \text { Distorsion de la vraie nature } \\
\text { de l'événement pour refléter } \\
\text { les valeurs du système } \\
\text { politique en vigueur. }\end{array}$ \\
\hline
\end{tabular}

Source: J.R. Brent Ritchic, Assessing the Impact of Hallnark Events: Conceptual and Research Issues, in Journal of Travel Research, vol. 23, no 1, Summer 1984, p. 4.

\section{Projets immobiliers directement associés au $350 \mathrm{e}$ anniversaire de Montréal}

En 1992, Montréal bénéficiera de plusieurs nouveaux ensembles immobiliers dont la réalisation ou la mise en chantier coilncide avec la décision de célébrer le 350 e anniversaire de Montréal. À long terme, certains seront tentés de dire que plusieurs de ces projets se seraient réalisés de toute façon. Toutefois, il ne faut pas sous-estimer le fait que, dans un contexte où les fonds ne sont pas disponibles pour répondre à tous les besoins, le choix de célébrer le 350 anniversaire a eu un poids important dans la prise de décision pour la réalisation de certains projets.

Résumons brièvement l'affectation de ces budgets:
Parc des îles

Pour une somme d'environ $55 \mathrm{M}$ \$, le gouvennement fếdéral fait un cadeau à la ville de Montréal d'un nouveau parc en milieu urbain, soit la partie ouest de l'île Ste-Hélène qui comprendra dorenavant une aire de spectacles en plein air, un lac et un point de vue extraordinaire sur Montréal.

\section{Biosphère}

De plus, le gouvernement fait cadeau à la Ville de Montréal de la restauration de la Biosphère et de l'aménagement d'un centre d'interprétation et de protection de l'environnement pour une somme totalisant plusde 17.5 M\$. Ces deux dons, pare desîles et biosphère, constituent globalement la contribution directe que fait le gouvernement fédéral à la Ville de Montréal pour 1992. 


\section{Centre d'interprétation}

\section{historique de Pointe-à-Callières}

Ce projet, bien que longuement souhaité par les intervenants associés à la mise en valeur du Vicux-Montréal, a été réalisé en tenant compte de la venue prochaine des célébrationsdu 350 e anniversaire. Totalisant près de $30 \mathrm{M} \$$, ce projet vise à mettre en Evidence le premier établissement et les premiers aménagements de Montréal. Doté d'outils didactiques importants et équipé des plus récentes techniques audio-visuelles, le Centre de Pointe-ả-Callières sera définitivement la porte d'entrée sur l'histoire de Montréal et la mise en évidence de son patrimoine.

Co-financé par le gouvernement du Québec, le gouvernement du Canada et la Ville de Montréal, la Pointe-à-Callières est définitivement un Equipement dont la réalisation est étroitement associće à la venue des célébrations du $350 \mathrm{e}$.

\section{Rénovation du Marché Bonsecours}

Fermé au public depuis très longtemps, le Marché Bonsecours redevient, en 1992, un lieuderencontre, d'expositionsetd'animation populaire. Dessommes totalisant plusde $5 \mathrm{M}$ \$ auront été consacrées à la rénovation partielle du bâtiment, à sa mise aux normes et à la préparation des exhibits qui y seront présentés. Les célébrations du 350 e permettent de redonner à la population et aux touristes un bátiment historique significatif qui contribuera de manière importante à relancer l'économie de services dans l'est du Vieux-Montréal: restaurateurs, boutiques et galeries d'art devraient normalement en profiter en 1992 et au cours des années subsiquentes.

\section{Biodôme/Parc olympique}

Ce projet, qui constitue en soi une première mondiale, est le cadeau du gouvernement du Québec à la Ville de Montréal dans le cadre des célébrations du $350 \mathrm{e}$. Bien que l'idée de réaliser ce projet ait été initiée indépendamment de la préparation des célébrations, l'année 1992 constitue la date significative qui a pernis de catalyser l'intérêt des décideurs à réaliser cet équipement dans les meilleurs délais.

Au total, près de $50 \mathrm{M} \$$ d'investissement, la création d'emplois permanents et l'ajout à Montréal d'un nouveléquipementoriginalet unique en son genre en Amérique du Nord. Les retombées économiques et sociales de cet equipement seront très importantes dès 1992.

\section{Parcs et places publiques}

L'administration municipale de Montréal a entrepris dess 1987 un vaste programme de restauration et de rénovation des espaces verts de Montréal. Bien que ceprogramme se réalise sur une période de plusieurs années, deux equipements, le Champ-de-Mars et le Square Berri sont en voie d'être complétés pour 1992. La réalisation prioritaire de ces deux projets visent a compléter des places publiques accessibles dans les quartiers du centre-ville. C'est dans la perspective des célébrations du 350 e que la décision a été prise de réaliser prioritairement ces deux équipements. Au total, plus de $10 \mathrm{M} \$$ sont consacrés à l'aménagementetă l'équipement de ces lieux qui deviendront de endroits fort fréquentés dans le cadre des célébrations.

\section{Projets immobiliers dont la réalisation coincide avec les célébrations du $350 \mathrm{e}$}

Depuis longtemps, Montréal attend un grand nombre d'équipements publics et ce, afin de compléter sa vocation de ville touristique. Connue comme ville d'ambiance et d'événements, Montréal deviendra, en 1992 , bénéficiaire de l'inauguration d'un grand nombre d'équipements qui ajouteront à la qualité de son produit touristique. Au total, plus de $200 \mathrm{M} \$$ de projets ont été réalisés au cours des demières années. La fin de ces travaux coilncide avec les célébrations du 350e. Rappelons brièvement chacun d'entre eux.

\section{Réalisation de la partie est et ouest du Vieux-Port de Montréal pour une somme totalisant $65 \mathrm{M} \mathrm{\$}$}

Attendu depuis près de 15 ans, le projet du Vieux-Port se réalise finalement. Bien qu'associé aux célébrations du 350 e, le projet du Vieux-Port est en soi un redéploiement des ressources et de l'aménagement de la partie sud du Vieux-Montréal.

\section{Agrandissement du Musée des Beaux-Arts}

Pour un investissement totalisant $80 \mathrm{M} \$$, le Musée des Beaux-Arts de Montréal devient enfin un musée de classe mondiale avec des espaces publics suffisants pour être intégré aux circuits des grandes expositions itinérantes.

\section{Rénovation et agrandissement du Musée McCord}

Moins connu, le Musée McCordest toutefois unique en son genre au Canada et, avec son agrandissement, il fera dorenavant partie du patrimoine international de Montréal: un investissement privé de plus de $30 \mathrm{M} \$$ consacréa lapréservation de notre héritageet de notre culture.

Le nouveau Musée d'Art Contemporain Longtemps souhaité, longtemps désiré, enfin réalisé, $38 \mathrm{M} \$$ investis au centre-ville de Montréal et ce, afin de mettre en évidence l'art actuel des artistes canadiens el québécois.

Le complexe et le musée de l'humour Projet privé de $21 \mathrm{M}$ \$ auquel sont associés les trois paliers de gouvernement, ce nouvel équipement contribuera à la relance économique dela rue Saint-Laurentausud de la rue Sherbrooke.

\section{Rénovation de bâtiments publics}

En complément des projets décritsci-dessus, la Ville de Montréal, de même que plusieurs promoteurs, ont entrepris la réalisation de nouveaux projets immobiliersouencore, tout simplement, la rénovation de certains bâtiments. Soulignons la rénovation du Palais de la civilisation pour une somme d'environ 10 M\$, la rénovation des lieux publics de l'hôtel de ville de Montréal, la restauration de la toiture du Vieux-Palais sur la rue NotreDame et, enfin, la réalisation de plusieurs projets privés tels le Centre de Commerce Mondial, la tour Lavallin-Téléglobeet, enfin, la tour IBM-Marathon.

Aucun des projets décrits dans cette demière section ne peut être associé directement aux célébrations du $350 \mathrm{e}$ mais, dans bien des cas, les dates d'inauguration et de fin des travaux coincident avec 1992. À ce titre, ils seront perceptuellement intégrés à l'héritage des célébrations du $350 \mathrm{e}$ anniversaire de Montréal. Au plan économétrique, il faudra toutefois être prudent et ignorer ces investissements dans tout calcul de retombés directes car cela ferait gonfler indûment les donnés réelles des célébrations du 350 .

\section{Les effets à court terme et long terme des célébrations du $350 \mathrm{e}$}

\section{A court terme}

A court terme, la contribution la plus significative descélébrations de 1992 sera d'avoir augmenté de manière significative le potentiel et 1'achalandage de Montréal comme destination touristique. En effet, 1992 sera une anné importante en terme d'accueil de congrès et d'événements associatifs. Pourquoi? Tout simplement parce que les Montréalais etles organisateurs d'evénementsont 
ciblé 1992 comme date d'accueil de leurs événements. Il en découle que Montréal recevra un nombre important de congrès et d'activités associés à la tenue de réunions d'entreprises.

Cette augmentation d'achalandage contribuera à confirmer larelance de l'industrie des congrès à Montréal et ce, après deux années difficiles, 1990 et 1991. Toutefois, il ne faut pas perdre devuequel'année 1992 constituera dans ce secteurd'activité la pointe supérieure d'un cycle qui, en général, évolue sur 7 à 8 ans. Encomplémentdesclientèlesdecongrès, Montréal accueillera certainement un nombre supérieur de visiteurs et de touristes, principalement du Canada, du Québecet des Etats-Unis, Cela est normal, compte tenu qu'en 1991-1992, les budgets consacrés à la promotion touristique doublerontetquel ${ }^{\dagger}$ offre de programmation des célébrations du $350 \mathrm{e}$ contribuera à faire de Montréal une destination alléchante.

Contrairement aux perceptions de plusieurs, l'Europe de 1992 , les olympiques et 1'Exposition de Séville ne nuiront pas substantiellementau partage des clienteles. Une nuance, toutefois: l'instauration denouvelles taxesdeconsommation et un climat politique incertain pourront contribuer à freiner cette augmentation.

D'autres bénéfices sont anticipess, à court terme: une augmentation de la durée de séjour, l'ouverture de nouveaux attraits touristiques et une notoriété médiatique conjoncturelle associées aux célébrations du $350 \mathrm{e}$ et a l'inaugurationde nouveaux bâtiments et sites prestigieux. De plus, l'accueil de visiteurs de marque contribuera à court terme à augmenter la notoriété de Montréal.

Enconclusion, à courtterme, il faut souligner doncquel'économie touristique de Montréal bénéficiera d'une performance supérieure à celle vécue au cours des trois dernières annees, à une augmentation du nombre de visiteurs et, par conséquent, du taux $d^{\dagger}$ cocupation et également à une augmentation des dépenses de consommation réalisées sur place.

A joutons ă la venue de touristes le fait que les Montréalais voyageront peut-être moins à l'étranger pour participer davantage aux célébrations et y engageront davantage d'argent dans le cadre de leurs activités de loisirs. Thériquement donc, lescommerçants et entreprises de services devraient également profiter en 1992 d'un regain d'activité.

\section{A long terme}

A long terme, les célébrations de Montréal contribueront, à mon avis, de manière significative, à transformer l'économie touristique de la région montréalaise. 1992 sera considérée comme une plaque toumante, un point de repère dans le temps à partir duquel on commencera à établir des comparaisons. Comme on a, par le passé, établi des comparaisons avant 1967 et après 1967, avant 1976 et après 1976, 1992 deviendra définitivement l'année bannière à partir de laquelle on commencera à comparer l'evolution du tourisme dans la région métropolitaine au cours de cette décennie.

1992 apportera des modifications profondes à l'offre touristique montréalaise. Tel que nous $1^{\prime} a v o n s$ décrit ci-dessus, le potentiel attractif pour développer de nouvelles clientèles seradorénavant présent. Cepotentiel, sous forme de produits nouveaux et de sites à visiter, permettra de modifier des comportements deconsommation et définitivementde prolonger la durée de séjour des visiteurs étrangers au Québec et à Montréal en particulier. L'inauguration d'un grand nombre de sites permettra de développer un positionnement stratégique différent et de compléter le repositionnement de Montréal en terme marketing de ville/événements ambiance à ville de produits, capable de concurrencer d'autres métropoles et destinations.

En effet, au cours des annces 1980, Montréal possédait une réputation enviable et un positionnement unique comme ville spécialisée dans l'organisation d'événements. Aujourd'hui, de nombreuses metropoles et destinations se sont $\hat{E}_{\text {quipésen conséquence }}$ et font, aujourd'hui, concurrence a Montréal au niveau de la qualité de vie et au niveau de l'organisation d'événements majeurs. Il en découle que l'arrivée à Montréal et l'ajout de produits muséologiques exclusifs, tel le biodôme, et de produits de fréquentation, tel le parc des îles, contribueront à consolider le positionnement stratégique de Montréal auprès des multiplicateurs, organisateurs de tourisme. Dorénavant, après 1992, Montréal possédera un biodồme unique au monde, un des plus grands parcs et espace verten milieu urbain, le parc des îles, un muscé des beauxarts de classe mondiale, un musbe d'art contemporain unique au Canada, des pares et places publiques au goût du jour, une collection d'art public novatrice, autant d'élements qui permettront de susciter de $1^{1}$ intếrêt auprès de journalistes multiplicateurs et éventuellement de visiteurs.
D'autre part, malgré ces ajouts importants, les succès de 1992 ne garantissent pas en soi le succès de Montréal comme destination touristique. En effet, une fois l'événement passé, il ne faudra pas se reposer sur ses lauriers et, au contraire, il faudra poser les gestes d'investissement requis pour faire la mise en marché de ce nouveau produit. Montréal a, par le passé, en 1967 et 1976, profité d'opportunités exceptionnelles. Malheureusement, dans les années qui ont suivi, le marketing touristique de Montréal a été un peu laissé pour compte. Il faut poursuivre les efforts systématiques entrepris au début des années 1980 et maintenir, après 1992 , le niveau de financement de la promotion et du développement de l'accueil touristique à Montréal. Sans ces deux leviers qui génèrent eux-mêmes des effets multiplicateurs, Montréal ne bénéficiera pas des retombées souhaitées par ceux et celles qui ont planifié l'organisation de ces célébrations dês 19861987.

\section{En toute objectivité}

En toute objectivité, on pourrait être très critiqueàl'Egard de certaines décisions prises quant à la pertinence de célébrer ou non en 1992 le 350 e anniversaire de Montréal. Toutefois, cette décision a été prise, les investissements ont été réalisés, une priorité a été donnée à la réalisation de certains equipements et il faut maintenant en profiter pour relancer et renforcer le développement de l'économie touristique du Québec. C'est. une industrie génératrice d'emplois, génératrice de circulation de biens et services, et qui supporte de nombreuses entreprises dans le secteur de la transformationet dans ledomaine alimentaire. Le véritableenjeu de 1992, c'est 1'après 1992, c'est-ä-dire le développement de la stratégie de communication et de promotion qui permettra à Montréal de bénéficier à long terme des retombées des célébrations du 350 e.

Au plan organisationnel, certains pourraient être critiques à l'égard du type d'événements réalisés, du type d'animation proposée, etc. Malgré tout, Montréal bénéficiera d'équipementsnouveaux, deplacespubliques restaurées et d'un Vieux-Montreal actualisé et capable d'accueillir de nombreux visiteurs. Autant d'opportunités qui permettront aux intervenants touristiques de stimuler leur créativitéet de convaincre éventuellement de nouvelles clientèles. 